\title{
Kinetic Monte Carlo Modeling of Graphene Sheet Growth During CVD
}

\author{
Hironori Kondo ${ }^{\dagger}$ \\ $\dagger$ Harvard College, Harvard University \\ 1954 Harvard Yard Mail Center, 1 Oxford Street, Cambridge, MA 02138 \\ Author email: hironorikondo92@gmail.com
}

\begin{abstract}
Graphene is a material of key interest across several research fields. Bulk graphene synthesis, however, has long remained a challenge for larger-scale projects and real-world manufacturability. This work seeks an improved understanding of graphene sheet growth via computational modeling, with the objective of maximizing grain size. To this end, the kinetic Monte Carlo method is used to simulate chemical vapor deposition under various configurations of carbon flow and graphene seeding. Ultimately, both quantitative and qualitative results are obtained to shed light on graphene growth mechanisms, with insights into real-world synthesis and future computational models.
\end{abstract}

\section{Introduction and Background}

In recent years, graphene has attracted much attention within the research community. In addition to its mechanical advantages, the material has demonstrated promising electrical properties when used in photovoltaics, ${ }^{1}$ hydrovoltaics, ${ }^{2}$ transistors, ${ }^{1,3}$ lithium-ion batteries, ${ }^{4}$ and pressureretarded osmosis systems, ${ }^{5}$ among other applications. Bulk synthesis of graphene hence becomes of high interest, both to realize larger-scale research projects and to enable real-world manufacturing.

One popular method of graphene synthesis is via chemical vapor deposition (CVD; see Appendix A for details) on copper $(\mathrm{Cu})$ subtrates, ${ }^{2,3,6,7}$ which have been found to yield more uniform monolayers than other substrates. ${ }^{1}$ The challenge, however, is in producing larger grain sizes via CVD, ${ }^{1,7}$ as this dictates the amount of usable, uniform graphene produced. Hence, a better understanding of graphene sheet growth is highly desired to expand the material's practical impact. ${ }^{1,8}$

To this end, this work computationally models graphene sheet growth during CVD on a $\mathrm{Cu}$ substrate, analyzing the effects of "pre-seeding" (Fig. 1) and carbon flow rate on grain size. At its most general, the model focuses on four forms of movement (henceforth "events") within a pre-defined hexagonal lattice: incoming carbon flow, surface diffusion, carbon attachment, and carbon detachment (Fig. 2). To simulate these events, we follow a four-step process: 
1) randomly seed carbon "atoms", 2) count the number of neighbors of each atom, 3) apply the kinetic Monte Carlo $(\mathrm{kMC})^{8,9}$ method, and 4) repeat the cycle across several iterations.
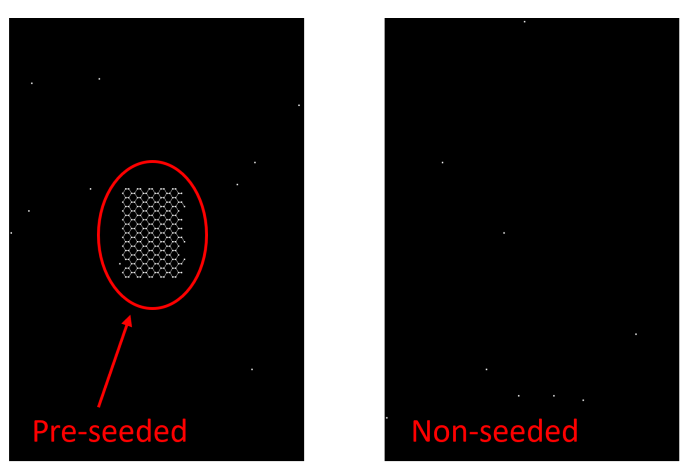

Figure 1. Comparison of pre-seeded (left) and non-seeded (right) samples.

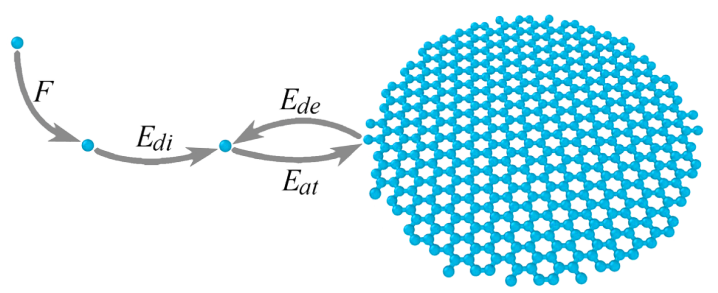

Figure 2. Schematic of all kMC events. F represents the flow rate of carbon atoms into the CVD chamber, $\mathrm{E}_{\mathrm{di}}$ diffusion along the substrate surface, $\mathrm{E}_{\text {at }}$ bond formation (i.e., attachment), and $\mathrm{E}_{\mathrm{de}}$ bond breakage (i.e., detachment). Adapted from Chen et al. 2020. ${ }^{8}$

We begin by randomly seeding carbon "atoms" into a simulated CVD chamber, modeled as a two-dimensional "box". This mimics the incoming flow of carbon, where the number of seeded "atoms" at each iteration corresponds to the physical flow rate. From there, we count the number of neighbors of each atom within the box, which reveals the number of possible events (e.g., surface diffusion, forming one bond, forming two bonds, etc.). From there, we can apply kMC, which relates the energy of each event to a corresponding probability determined by the Boltzmann distribution. Based on these probabilities, one particular event is chosen for each atom, completing one "iteration" of the simulation (details of this calculation are expanded upon in Appendix $\mathrm{B}$ ). The entire process is then repeated until the desired number of carbon atoms has been introduced. Hence, we are able to simulate the movement of individual atoms based on the energy required for each event, which affects the probability of said event occurring.

For our $\mathrm{Cu}$ substrate specifically, these necessary energies are well-documented in the existing literature. In particular, we use values derived from first principles by $\mathrm{Wu}$ et al. $2015 .{ }^{10}$ Critically, we make four major approximations: 1) all edge diffusion is disregarded; 2) all dimer movement is disregarded, making single-atom events the only source of movement; 3) the event energies are proportional to the number of bonds being formed or broken, independent of the domain geometry; and 4) the graphene is strictly a monolayer, with the simulation restricted to two dimensions and no "stacking" of atoms permitted. Approximations one through three are visualized below in Figures 3 to 5, which are adapted from Chen et al. $2020 .^{8}$

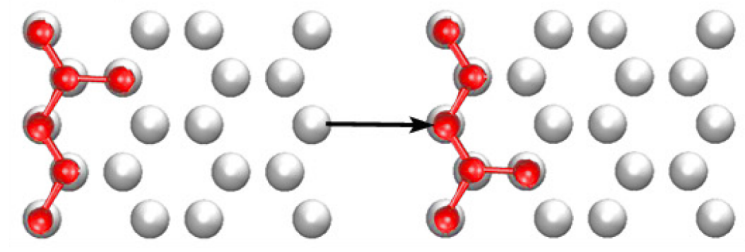

Figure 3. A visualization of edge diffusion, wherein the carbon atom moves along the edge of a graphene domain in a single event. We instead approximate this by breaking the bond and forming a new one in two separate events. 


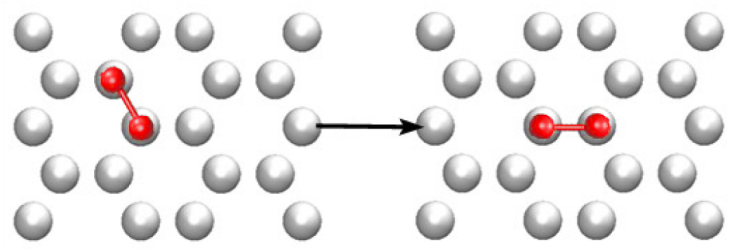

Figure 4. A visualization of dimer rotation. This is not possible within our model; for such a movement to occur, the dimer must break apart, and the atoms must move independently to form a new bond in the new position.

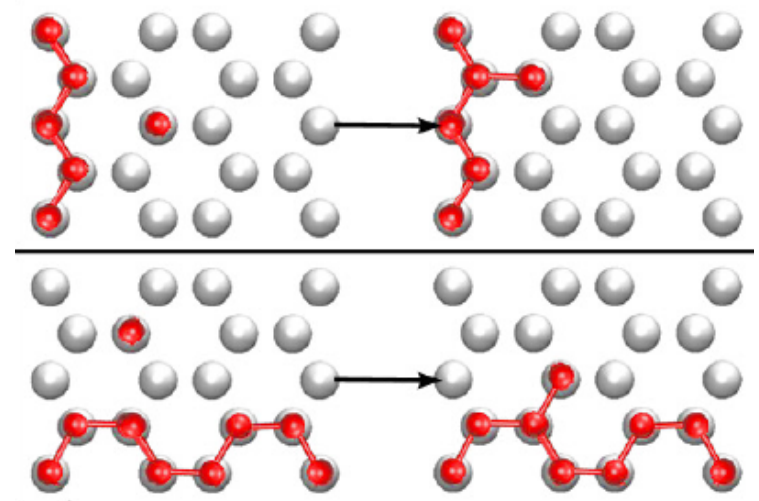

Figure 5. A visualization of carbon atoms binding to different domain geometries. From first principles, these two events have different energies, due to the differences in the surfaces the atoms are attaching to. We approximate these two events to be the same, based on the fact that only one bond is forming.

To conduct our analysis, we implemented the above procedure in MATLAB R2021a with the following parameters:

- Chamber size: 100 by 100 pixels

- Chamber temperature: $1000{ }^{\circ} \mathrm{C}$ (derived from existing literature ${ }^{1,8}$ )

- Possible atom locations: 5000 in total (based on the pre-defined hexagonal lattice)
- Initial configuration: Pre-seeded, non-seeded (conducted identically besides the presence or omission of a pre-seed)

- Flow rate: $2^{0}, 2^{1}, 2^{2}, 2^{3}, 2^{4}, 2^{5}, 2^{6}$, $2^{7}, 2^{8}, 2^{9}$ atoms per iteration (10 total variations of flow rate)

- Total number of atoms introduced: 2048 (sc. the number of iterations for each flow rate was different, and each "run", or "cycle", of the simulation ended when 2048 atoms were reached)

- Number of cycles per flow \& seeding variation: 500 (i.e., each unique configuration was run 500 separate times; each run was comprised of several iterations and ended when 2048 atoms were introduced)

These parameters made for 500 independent runs $\times 10$ different flow rates $\times$ two different initial configurations, totalling 10,000 independent trials (see Appendix $\mathrm{C}$ for details on the MATLAB implementation). With this information, we can determine how flow rates affect graphene grain size for both pre-seeded and non-seeded cases. Quantitatively, this was done by taking the arithmetic mean of each result across all 500 of its runs, then plotting the changes in grain sizes and grain counts (i.e., the number of individual graphene grains). For qualitative analysis, we converted the runs into images of graphene lattices for visualization. Finally, we abstracted the findings from both analyses to the overall grain size distributions, providing a better big-picture view of the simulation results. 


\section{Results and Discussion}

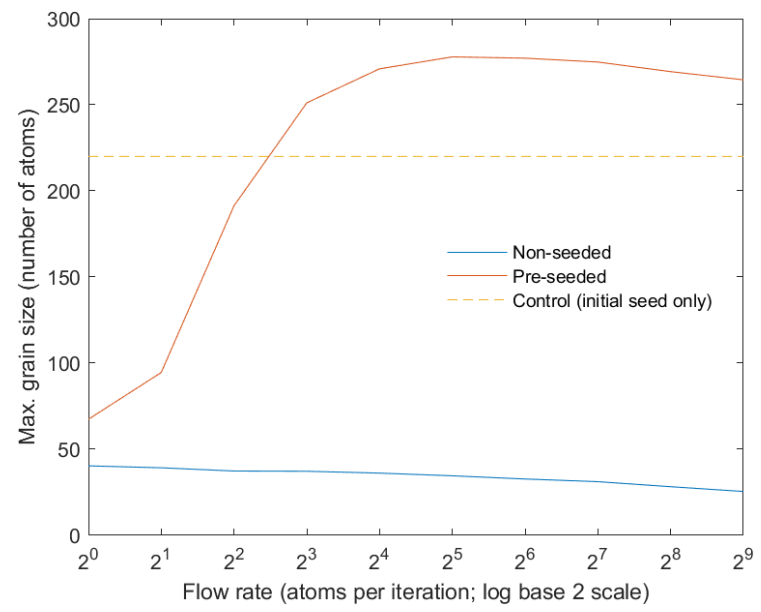

Figure 6. Mean maximum grain size by carbon flow rate, with a control (the atom count of the initial seed).

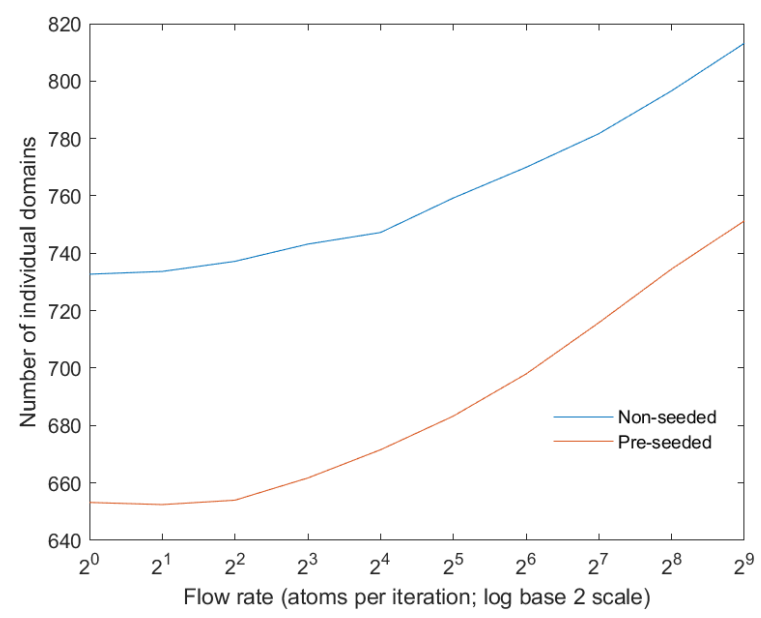

Figure \%. Mean grain count (number of individual grains) by carbon flow rate.

We begin with our quantitative results. Figures 6 and 7 show how mean maximum grain size and mean grain count vary by flow rate, respectively (reprinted larger in Appendix D). These results shed light into the mechanisms at play during graphene sheet growth.

For Fig. 6, we observe that the preseeded samples have consistently higher grain sizes than the non-seeded samples. In particular, the trends in the two clearly diverge: the pre-seeded samples are shown to increase and then decrease in grain size, while the non-seeded ones strictly decrease. Hence, in seeking larger graphene sheets, seeding is essential to 1) producing larger grains and 2) enabling significant increases in growth by controlling the flow rate.

We observe, however, that the preseeded samples do not always result in graphene growth. In particular, our first measured instance of growth was at a flow rate of $2^{3}$ atoms/iteration; every sample below this flow rate resulted in sheet shrinkage. A potential explanation for this phenomenon is that, due to the higher number of iterations necessitated by the lower flow rates, the atoms in the initial sheet are given more opportunities to diffuse away, leading to an overall decrease in grain size.

We also note that, for the pre-seeded samples, grain size does not strictly increase with flow rate. Instead, there is a local maximum at $2^{5}$ atoms/iteration, after which the grain size gradually tapers off. This phenomenon may again be explained by diffusion; instead of the sheet fragmenting however, the now lower number of iterations are not granting newlyseeded atoms enough opportunity to diffuse towards the sheet and bind to it. In essence, the graphene sheet becomes surrounded by loose carbon atoms that have not diffused and gathered into concrete grains.

This finding is supported by Fig. 7, wherein the number of grains increases with flow rate for both the pre-seeded and non-seeded cases. Hence, our notion that the number of loose atoms increases with flow rate is supported, as the total number of seeded atoms is consistent (i.e., 
2048), but the number of grains is increasing. As a note, we can also observe that the pre-seeded configurations have much fewer grains than the non-seeded ones, which aligns with what Fig. 6 has indicated about maximum grain sizes ( $s c$.

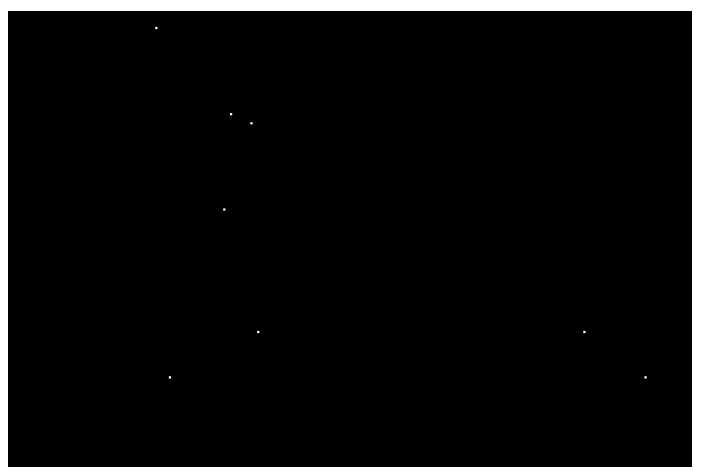

Figure 8. Non-seeded run with a flow rate of 8 atoms/iteration after one iteration.

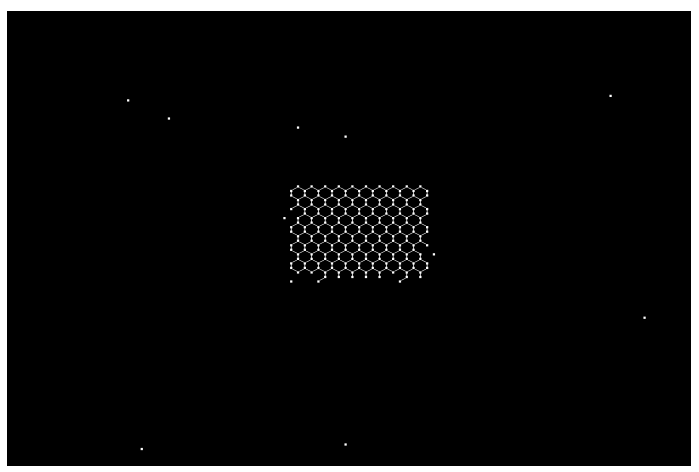

Figure 10. Pre-seeded run with a flow rate of 8 atoms/iteration after one iteration.

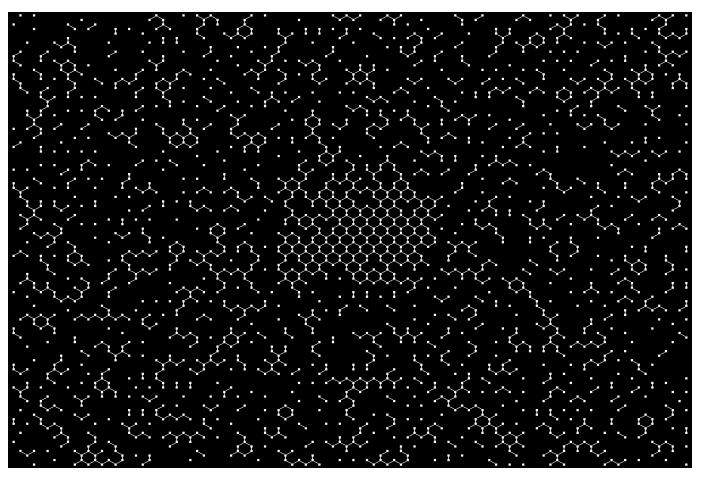

Figure 12. Pre-seeded run with a flow rate of 32 atoms/iteration after 64 iterations. for the pre-seeded samples, there are more atoms within a single grain and hence fewer "loose" atoms).

All of these observations and explanations are reflected in our qualitative analysis, summarized below in Figures 8 to 13 :

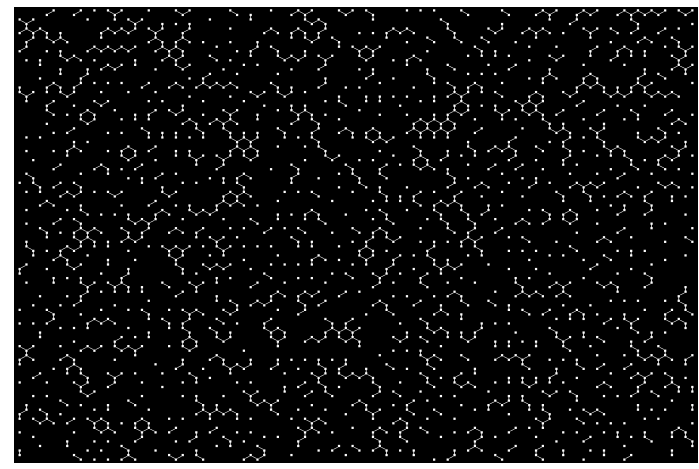

Figure 9. Non-seeded run with a flow rate of 8 atoms/iteration after 256 iterations.

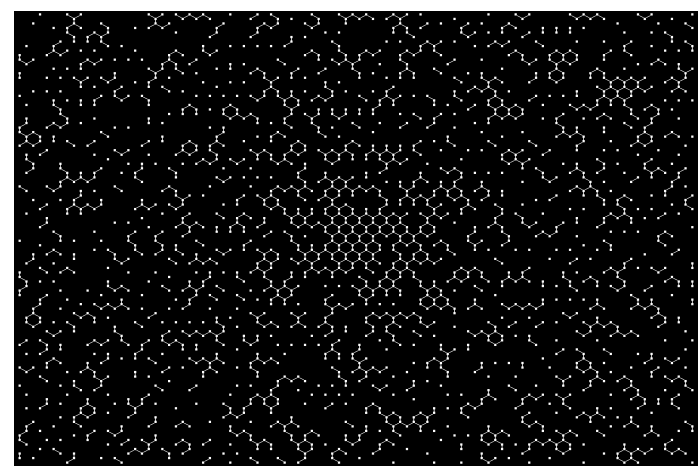

Figure 11. Pre-seeded run with a flow rate of 8 atoms/iteration after 256 iterations.

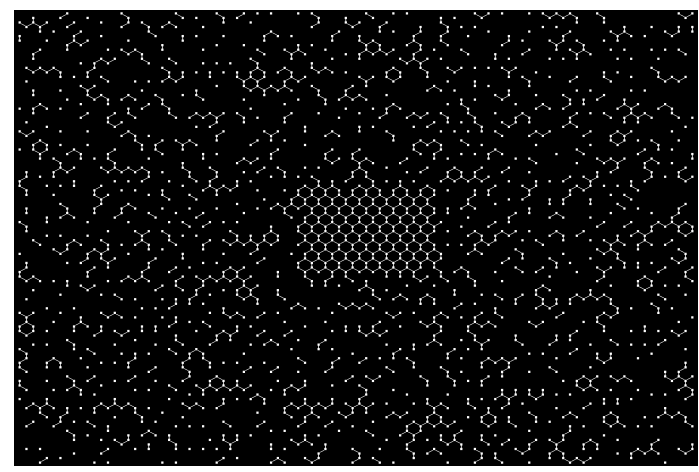

Figure 13. Pre-seeded run with a flow rate of 512 atoms/iteration after 4 iterations. 
These visualizations support the two overarching notions from our quantitative analysis:

1. Pre-seeding is instrumental to developing larger graphene grains, as the lack of a pre-seed creates smaller grains (Fig. 6) and scattered carbon atoms (Fig. 7). For instance, we see how a pre-seeded sample yields a denser congregation of atoms towards the middle (Fig. 11), whereas a non-seeded sample becomes sparse and fragmented (Fig. 9), despite the same flow conditions in both cases.

2. Diffusion (which is related to flow rate) controls the interplay between sheet fragmentation and growth (Fig. $6)$. We observe that, at a low flow rate, an initially rectangular pre-seed (Fig. 10) becomes more sparse (Fig.
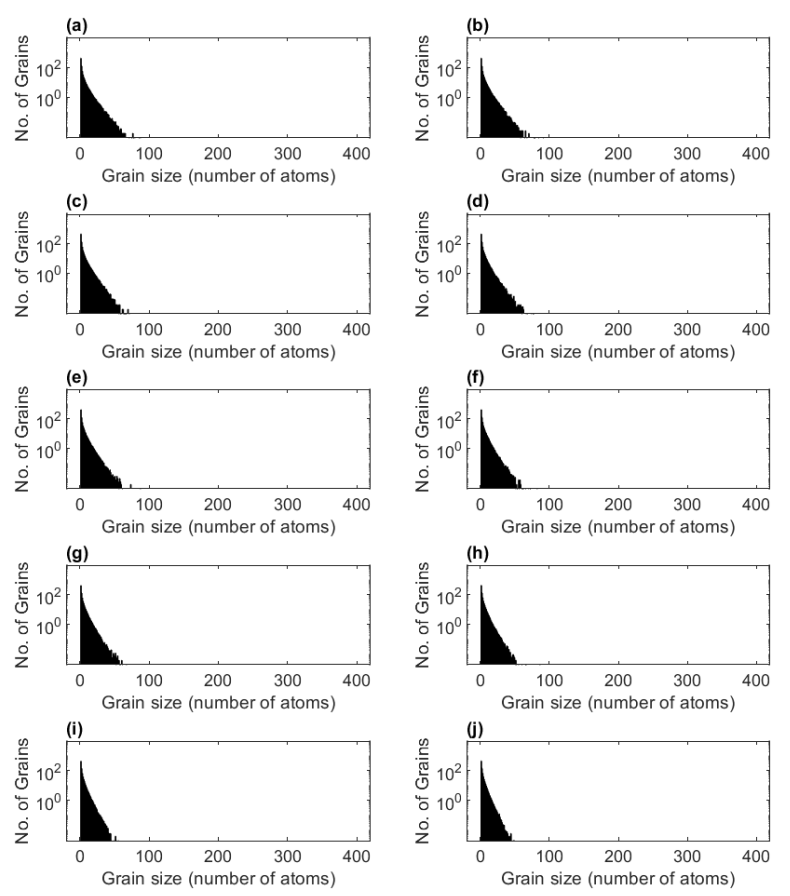

Figure 14. Histogram of mean non-seeded grain size distribution, for flow rates of (a) $2^{0}$, (b) $2^{1}$, (c) $2^{2}$, (d) $2^{3}$, (e) $2^{4}$, (f) $2^{5}$, (g) $2^{6}$, (h) $2^{7}$, (i) $2^{8}$, (j) $2^{9}$ atoms per iteration.
11), indicating an outward diffusion of atoms. If we increase the flow rate, this outward diffusion is reduced and the sheet ends up larger (Fig. 12); increase the flow rate too much, however, and the product ends up looking remarkably similar to the pre-seed (Fig. 13). In essence, the atoms are not given opportunities to flow towards the largest grain and cause it to grow. Hence, a delicate balance must be struck, wherein atoms are given opportunities to bind to the grain but not diffuse outward by too much.

Hence, our observations are consistent between both the quantitative and the qualitative results. To further extend these findings, we can abstract them to their effects on the overall distribution of grain sizes (reprinted larger in Appendix D):
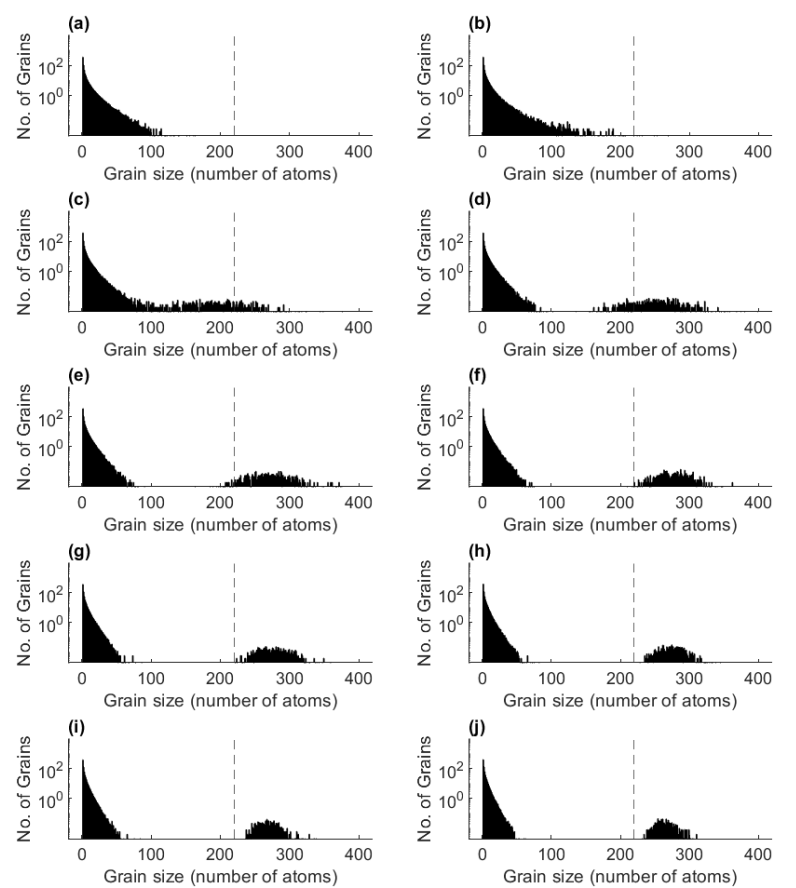

Figure 15. Histogram of mean pre-seeded grain size distribution. Dashed lines indicate initial pre-seed size. Refer to Fig. 14 for the respective flow rates of the subplots. 
Here, we have two key observations, which are taken in parallel to our previous two points:

1. The pre-seeded samples form a distinct "bump" in their distributions at higher grain sizes (Fig. 15). This is a reflection of our previous notion that pre-seeding is essential to creating larger-grain graphene sheets.

2. Higher flow rates cause horizontal compressions in the distributions, making for sharper, cleaner peaks (Fig. 14, 15). For the pre-seeded samples, lower flow rates do not yield distinguishable/sharp "bumps" in the higher-grain-size region (Fig. 15). These both reveal the role of diffusion, as previously described:

The horizontal compressions suggest that higher flow rates create less room for variability in the grain size distribution. This in turn suggests that fewer opportunities (i.e., iterations) are provided for atom movement, hence yielding a more fragmented product characterized by more small-size grains. Previously, we observed the same phenomenon in Fig. 7, 13.

The formation of the high-grainsize "bump" at high flow rates suggests that, at low flow rates, even the presence of a seed results in smaller final grains. This implies that the initial seeds fragment and disperse during the CVD process, a symptom of too much atom movement (i.e., too many iterations that permit too much outward diffusion). We previously observed the same phenomenon in Fig. 6, 11.
Hence, we observe that our quantitative and qualitative conclusions are reflected within the overall distributions of grain sizes. We thus have three distinct perspectives from which the same major findings can be derived, strengthening the overall case for our core notions.

\section{Conclusion and Outlook}

We have thus determined that pre-seeding is instrumental to grain growth, and flow rate controls the interplay between fragmentation (i.e., outward diffusion) and binding (i.e., inward diffusion and bond formation). Critically, we saw that lower flow rates create more opportunity for diffusion, leading to sparser products; higher flow rates, meanwhile, limit the amount of change from the initial seed, hence making for a less productive process. A balance between the two must be struck to maximize grain size, as depicted in Fig. 6. These findings are supported by both quantitative and qualitative analyses, and they are reflected in the overall grain size distributions of our samples.

The approximations of our model lead to limitations that should be addressed in future works. In particular, all samples show a high number of individual grains (Fig. 7), most of which are very small (Fig. $14,15)$. As this trait is not entirely consistent with the existing literature, ${ }^{8}$ the omissions of dimer movement and edge diffusion may be limiting the ability of the carbon atoms to congregate and form larger, uniform sheets. Future models should attempt to incorporate these kMC events, but implementation will be more involved.

In addition, while this work addressed CVD on $\mathrm{Cu}$ films specifically, its core structure can be adapted to other graphene synthesis methods. These include CVD 
on other metal films, non-monolayer processes, and other technologies such as Molecular Beam Epitaxy. Hence, in short, the conclusions herein yield insight into not only the mechanisms of graphene sheet growth, but also expandability to other synthesis processes and more thorough models of atom movement.

\section{Acknowledgements}

The author thanks Alvin Hsu, Liu Group, Harvard University Department of Chemistry and Chemical Biology (CCB) for advising this work. The author also thanks Senior Preceptor Lu Wang, Professor Kang-Kuen Ni of Harvard CCB and members of the PS 10 teaching staff for their guidance and supervision.

\section{References}

(1) Zhang, Y.; Zhang, L.; Zhou, C. Review of Chemical Vapor Deposition of Graphene and Related Applications. Acc. Chem. Res. 2013, 46, 2329-2339, DOI: $10.1021 / \operatorname{ar} 300203 n$.

(2) Zhang, Z.; Li, X.; Yin, J.; Xu, Y.; Fei, W.; Xue, M.; Wang, Q.; Zhou, J.; Guo, W. Emerging hydrovoltaic technology. Nature Nanotech 2018, 13, 1109-1119, DOI: 10.1038/s41565-018-0228-6.

(3) Li, X.; Cai, W.; An, J.; Kim, S.; Nah, J.; Yang, D.; Piner, R.; Velamakanni, A.; Jung, I.; Tutuc, E.; Banerjee, S. K.; Colombo, L.; Ruoff, R. S. Large-Area Synthesis of High-Quality and Uniform Graphene Films on Copper Foils. Science 2009, 324, 1312-1314, DOI: 10. 1126/science.1171245.

(4) Kucinskis, G.; Bajars, G.; Kleperis, J. Graphene in lithium ion battery cathode materials: A review. Journal of Power Sources 2013, 240, 66-79, DOI: 10.1016/j.jpowsour.2013.03.160.
(5) Jia, Z.; Wang, B.; Song, S.; Fan, Y. Blue energy: Current technologies for sustainable power generation from water salinity gradient. Renewable and Sustainable Energy Reviews 2014, 31, 91100, DOI: 10.1016/j.rser.2013.11.049.

(6) Xu, S.; Zhang, L.; Wang, B.; Ruoff, R. S. Chemical vapor deposition of graphene on thin-metal films. Cell Reports Physical Science 2021, 2, 100372 , DOI: $10.1016 /$ j.xcrp.2021.100372.

(7) Gao, L.; Guest, J. R.; Guisinger, N. P. Epitaxial Graphene on $\mathrm{Cu}(111)$. Nano Lett. 2010, 10, 3512-3516, DOI: 10 . 1021/nl1016706.

(8) Chen, S.; Gao, J.; Srinivasan, B. M.; Zhang, G.; Sorkin, V.; Hariharaputran, R.; Zhang, Y.-W. An all-atom kinetic Monte Carlo model for chemical vapor deposition growth of graphene on $\mathrm{Cu}(1$ 1 1) substrate. 2020, 32, 155401, DOI: 10.1088/1361-648X/ab62bf.

(9) Voter, A. F. In Radiation Effects in Solids, ed. by Sickafus, K. E.; Kotomin, E. A.; Uberuaga, B. P., Springer, Dordrecht: 2007, pp 1-23, DOI: 10.1007/ 978-1-4020-5295-8_1.

(10) Wu, P.; Zhang, Y.; Cui, P.; Li, Z.; Yang, J.; Zhang, Z. Carbon Dimers as the Dominant Feeding Species in Epitaxial Growth and Morphological Phase Transition of Graphene on Different $\mathrm{Cu}$ Substrates. Phys. Rev. Lett. 2015, 114, 216102, DOI: 10.1103/PhysRevLett. 114.216102 . 


\section{A CVD Fundamentals}

This work assumes a basic understanding of the CVD process; the purpose of this section is to fill in the fundamentals necessary to understand the computation model. For a more detailed look at CVD synthesis of graphene on metal film substrates, please refer to Zhang et al. 2013. ${ }^{1}$

At its most basic, CVD graphene synthesis is comprised of three elements:

1. A gaseous carbon source (e.g., $\mathrm{CH}_{4}$ )

2. A heated chamber (typically tubular)

3. A substrate

The carbon source is introduced into the chamber at a certain rate, described in our model as the "flow rate". In the heat of the chamber, the gas decomposes to yield pure carbon, which deposits onto the substrate. A graphene sheet can thus begin to form.

The purpose of the substrate is to provide a surface onto which the carbon can attach. It also behaves as a catalyst to lower the energy barrier of graphene formation, along with dictating the deposition mechanism. ${ }^{1}$ Within our model, this manifested as the discretization of the chamber (sc. by pre-defining a hexagonal lattice), as well as the specific event energies used within the kMC portion.

Though elementary, this information is sufficient to understand our model. It is by no means exhaustive in terms of graphene synthesis, and it certainly does not cover all forms and applications of CVD. Instead, it merely serves to contextualize the methodology of this work.

\section{B Kinetic Monte Carlo}

Previously, this work outlined the core tenets of the kinetic Monte Carlo method. The purpose of this section is to elaborate in further detail the specific mechanisms of $\mathrm{kMC}^{8,9}$ as it relates to our model.

The basis of kMC lies in energy. Carbon atoms interacting with the $\mathrm{Cu}$ substrate and with each other require energy; different interactions, or "events", require different amounts of energy, which can be derived from first principles. ${ }^{10}$

$\mathrm{kMC}$ begins by relating each event $i$ to a given rate $k_{i}$, related to the event's energy $E_{i}$ via the Boltzmann distribution

$$
k_{i}=\nu \exp \left(-\frac{E_{i}}{k_{b} T}\right),
$$

wherein $\nu$ is the frequency of atomic vibration (here $1.0 \times 10^{12} \mathrm{~s}^{-1}$ as per existing literature $\left.^{8}\right), k_{b}$ is the Boltzmann constant, and $T$ is the chamber temperature (here chosen to be $1000{ }^{\circ} \mathrm{C}$, again as per existing literature $\left.^{1,8}\right)$. The sum of all possible rates for a given atom at a given iteration is denoted by $k_{t o t}$.

These rates are used to weight a random number generator, such that the generator selects events with probabilities corresponding to the events' rates. To do so, we take the rates $k_{1}, \ldots, k_{M}$ of all possible events $1, \ldots, M$ for a given atom and stack them end-to-end in an array, of which we can then take the cumulative sum (sc. the first entry is $k_{1}$, the second is $k_{1}+k_{2}$, and the $h^{\text {th }}$ is $k_{1}+\ldots+k_{h}$ ). By drawing a random number $r$, we can select the first event $h$ at which the partial sum is less than or equal to $r k_{\text {tot }}$ (i.e., $h$ is the greatest element of $\{1, \ldots, M\}$ for which $\left.\left(k_{1}+\ldots+k_{h}\right) \leq r k_{t o t}\right)$. This event $h$ is chosen to occur, and the algorithm selects events with the correct probabilities as determined by the Boltzmann distribution. 
This algorithm is visualized below in Fig. 16. Note that, in our model, we determined the possible events $1, \ldots, M$ of each atom at each iteration by counting its neighboring atoms, along with each of their respective neighbors (see Appendix C).

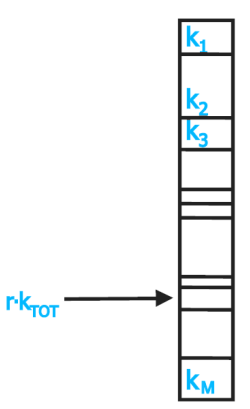

(a)

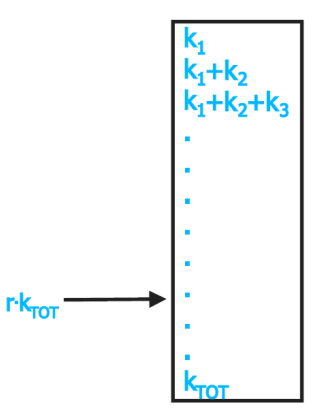

(b)
Figure 16. Visualization of the $k M C$ process. (a) Possible events are assigned rates corresponding to their probabilities, here depicted as length. A random number $\mathrm{r}$ is drawn, and $\mathrm{k}_{\mathrm{tot}}$ is the total sum of the rates (i.e., $\mathrm{k}_{1}+\ldots+\mathrm{k}_{\mathrm{M}}$ ). (b) Comparing $\mathrm{rk}_{\text {tot }}$ to the cumulative sums "weights" the random number generator to select events with the correct probabilities. Adapted from Voter 2007.9

Now, we have covered the fundamentals of how kMC selects events. There is a secondary portion that assigns time lengths to each event, again related to a weighted random number generator. For the purposes of this work, however, comparing across atom counts instead of time yielded more concrete insights, as doing so provides more standardization with which to understand grain sizes. Hence, this time-selection algorithm has been omitted, but there is some relevance to our model, as the total simulation time is related to the number of iterations as discussed herein. For details about this time algorithm, refer to Voter $2007^{9}$ and Chen et al. 2020. ${ }^{8}$

\section{MATLAB Code}

This section contains the MATLAB R2021a code that runs the simulations. It is a brief, reference-only outline of the model and by no means a complete exposition to its workings.

The code consists of two main elements: a live script that processes all 10,000 runs and gathers information about them, along with a function stored in a standard script that handles each individual run (which consists of several iterations). The rough breakdown is as follows:

- Live script

1. Initialize the variables

2. Track the indices of adjacent lattice points (i.e., neighboring positions)

3. Define a vector to map the lattice to a single-column vector

4. Run 500 non-seeded cycles of the simulation (by calling the function)

5. Gather pixel clusters per cycle

6. Sort the information into arrays

7. Repeat steps 4-6 for pre-seeded samples

8. Gather information from the initial pre-seed (as a control)

9. Export the workspace variables

- Function

1. Gather the input variables \& define the physical constants

2. Randomly seed atoms

3. Count neighbors for each atom

4. Run the kMC algorithm

5. Output the final CVD "box"

We begin by showing the live script, followed directly by the function: 


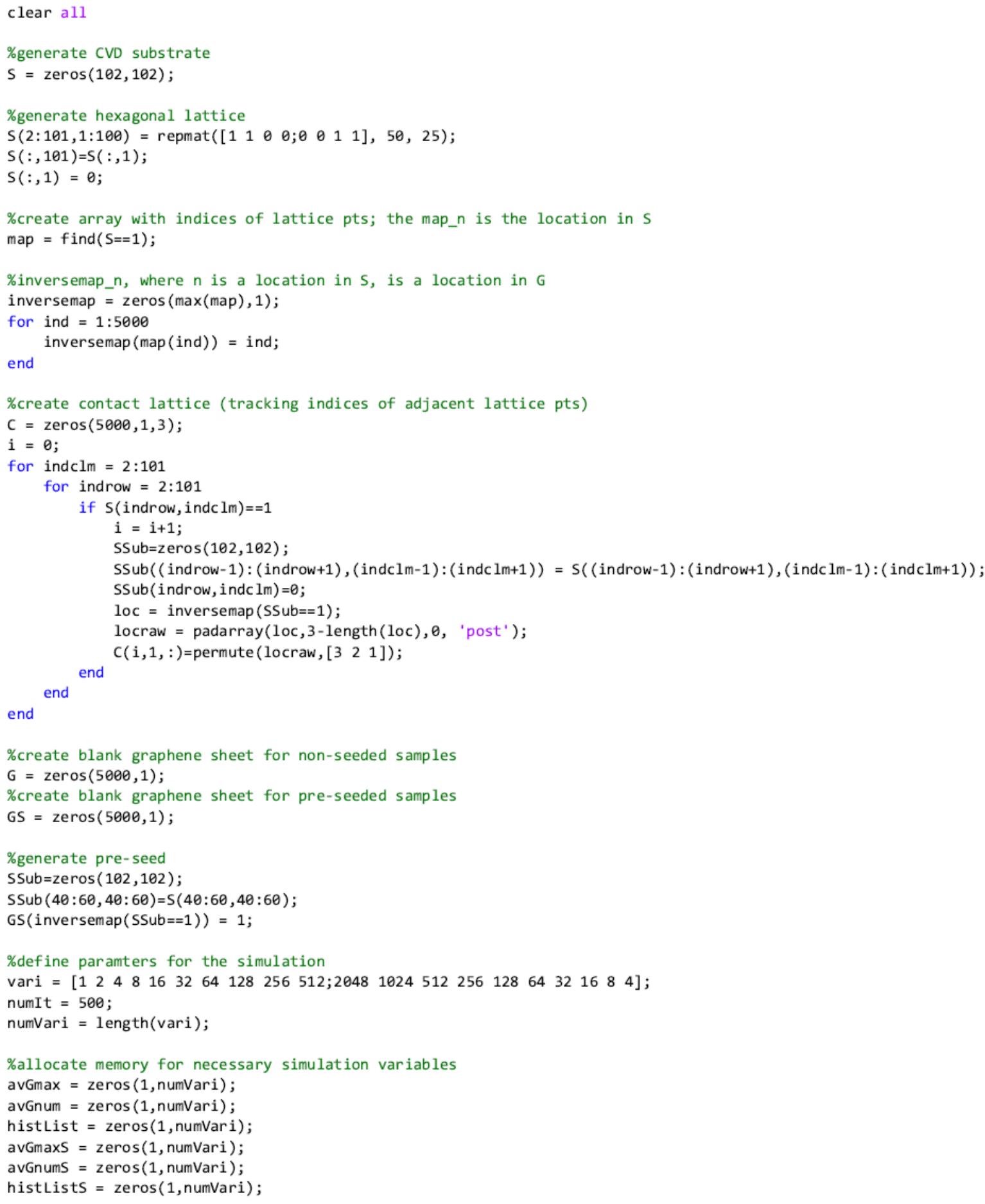

Figure 17. Code for setting up the live script workspace, including defining the hexagonal lattice, tracking adjacent lattice points, allocating memory for variables, defining simulation parameters, etc. 


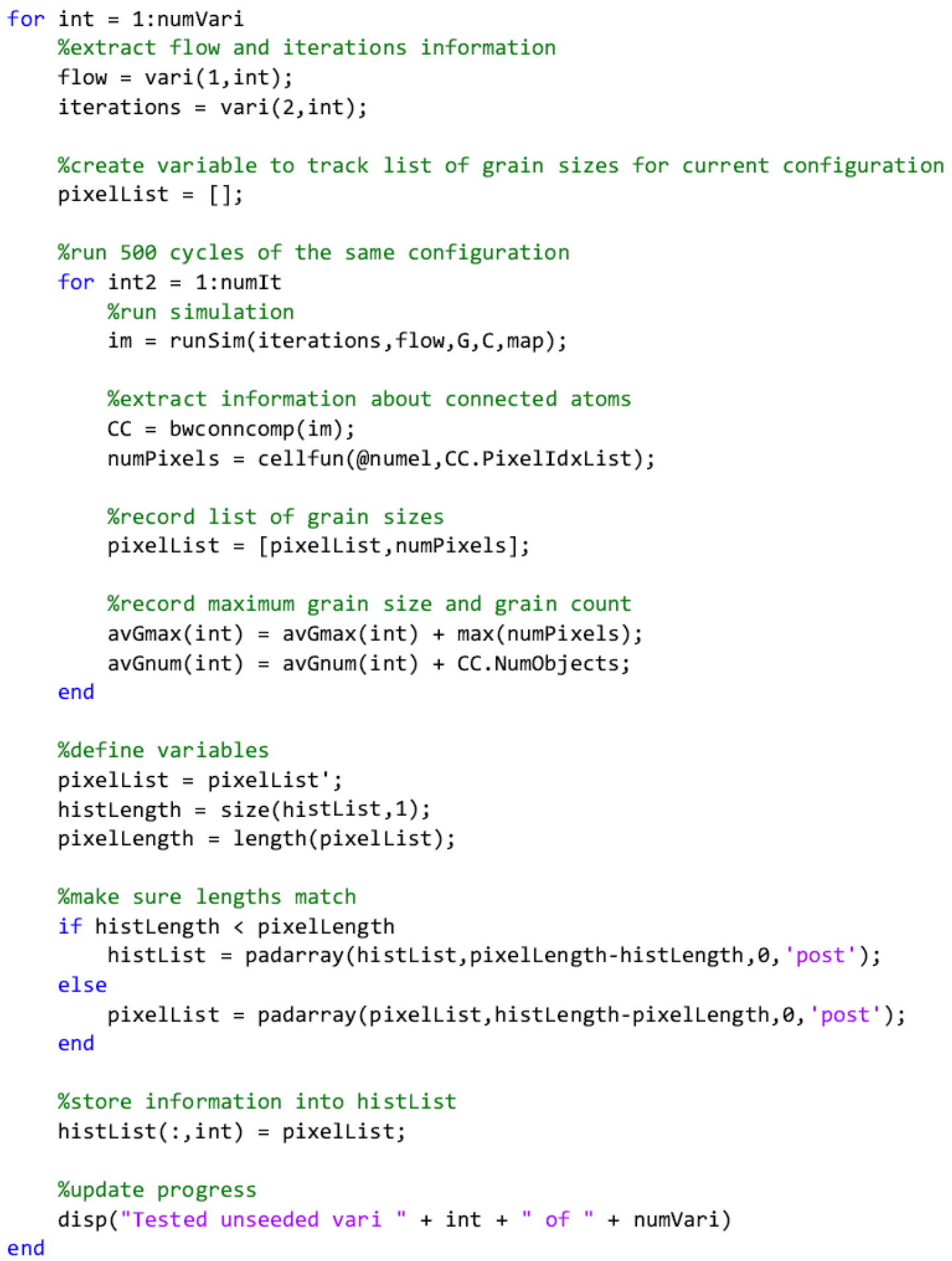

Figure 18. Code for running 500 non-seeded cycles of the simulation and gathering the relevant information about them for quantitative analysis. 


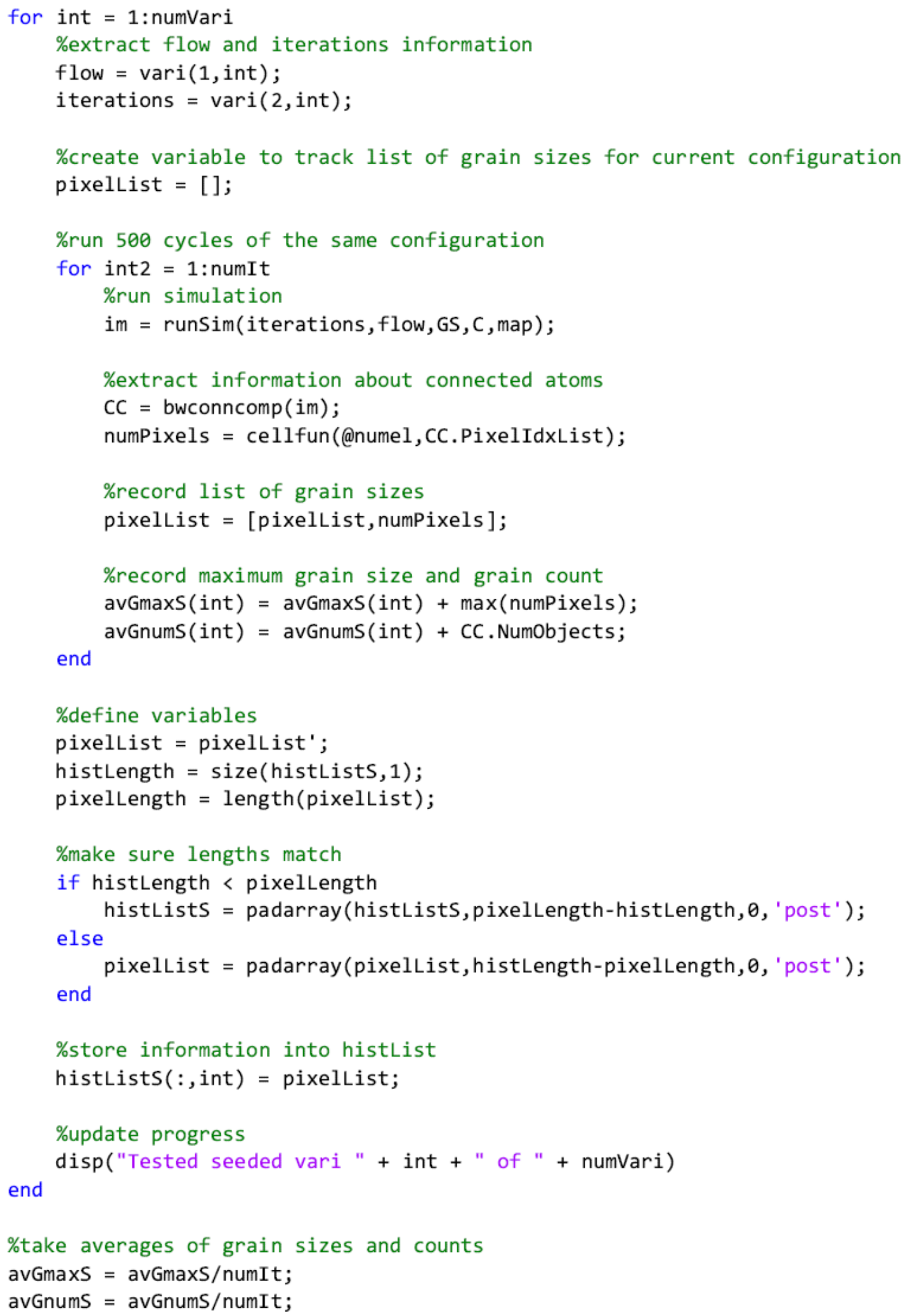

Figure 19. Code for running 500 pre-seeded cycles of the simulation and gathering the relevant information about them for quantitative analysis. 


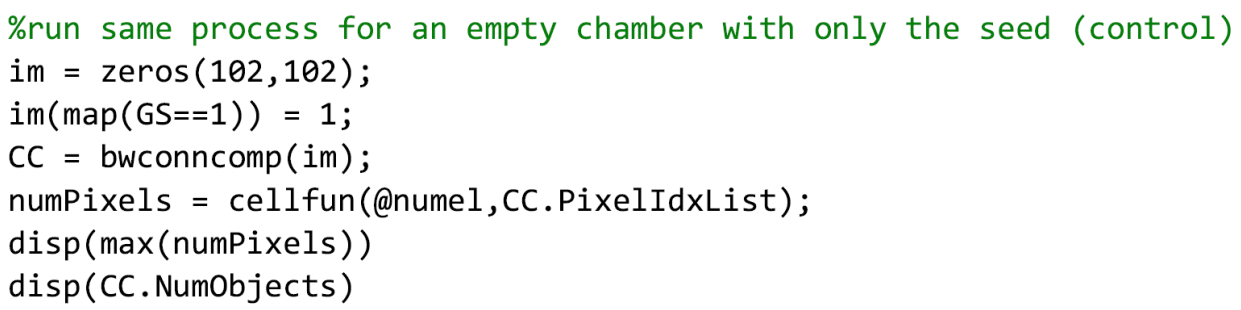

Figure 20. Code for extracting relevant information from the initial pre-seed (as a control, used in Fig. 6, 15).

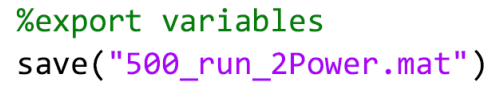

Figure 21. Code for exporting all workspace variables, such that they can be stored and used to make figures without re-running the live script.

This concludes the live script. Next is the function that runs each cycle:

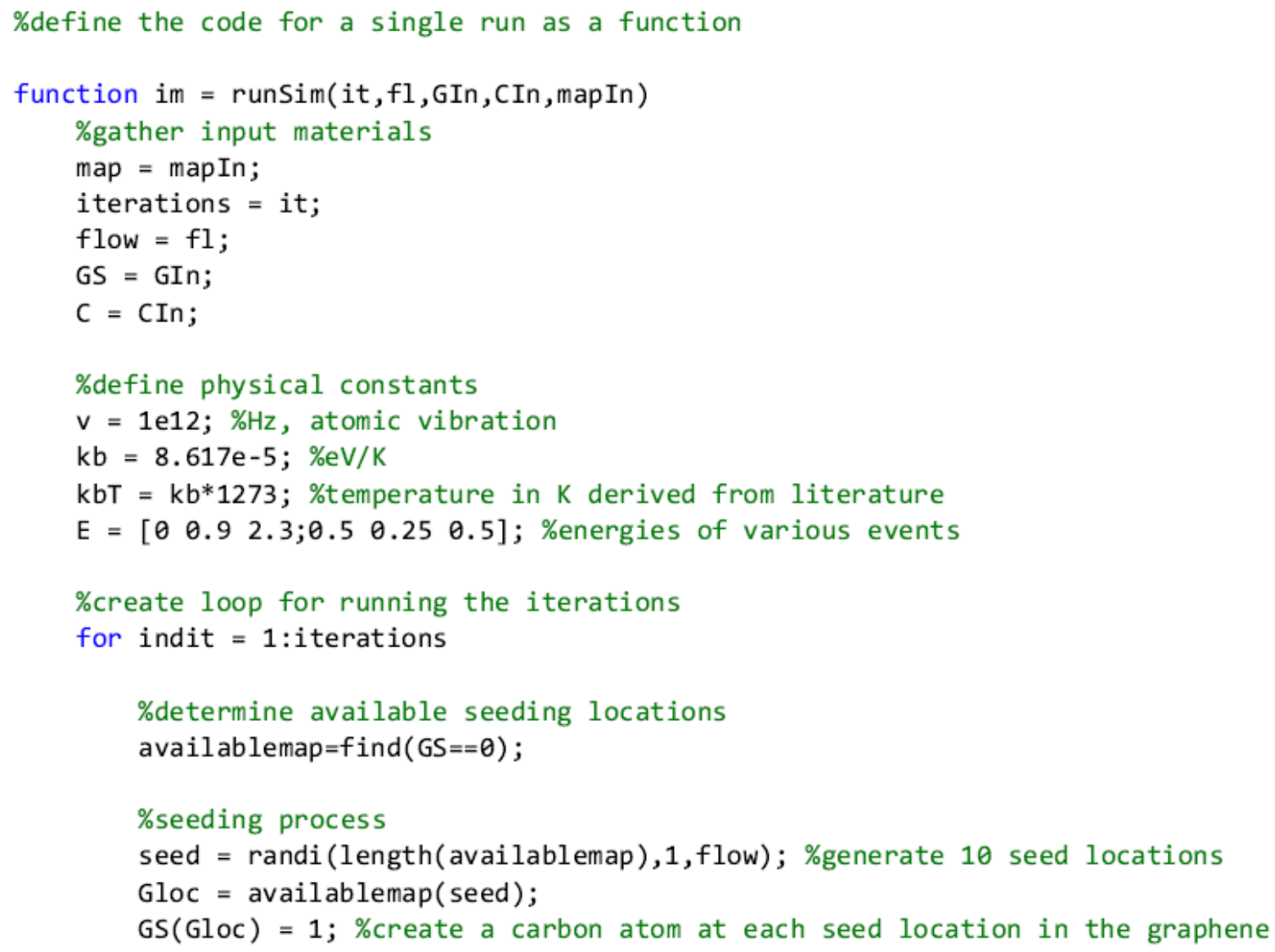

Figure 22. Code for extracting function inputs, defining physical constants, beginning the iterations, and seeding new carbon atoms. 


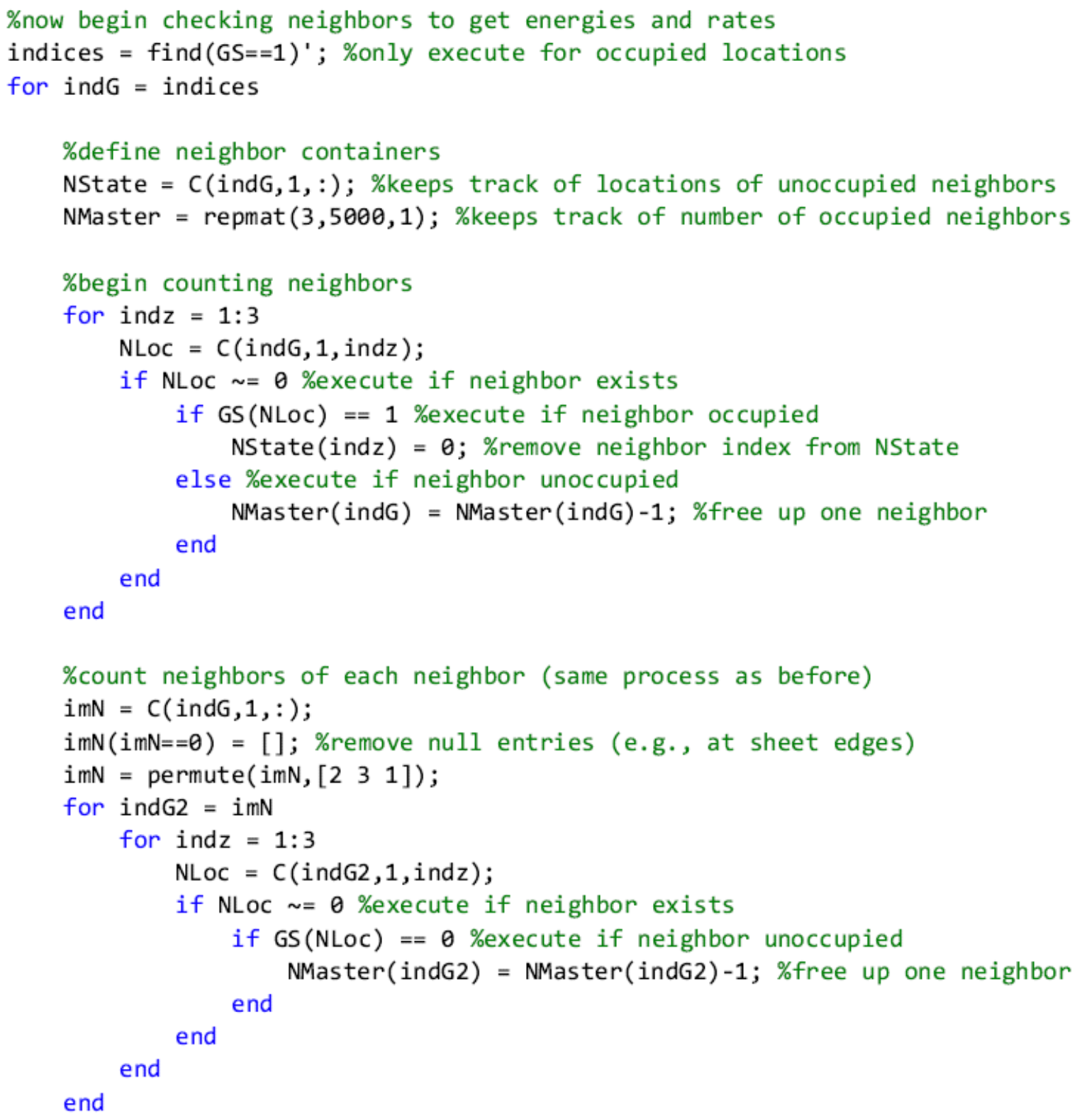

Figure 23. Code that checks for the neighbors of each existing atom, along with each of the neighbor's neighbors. These are later used to run the $k M C$. 


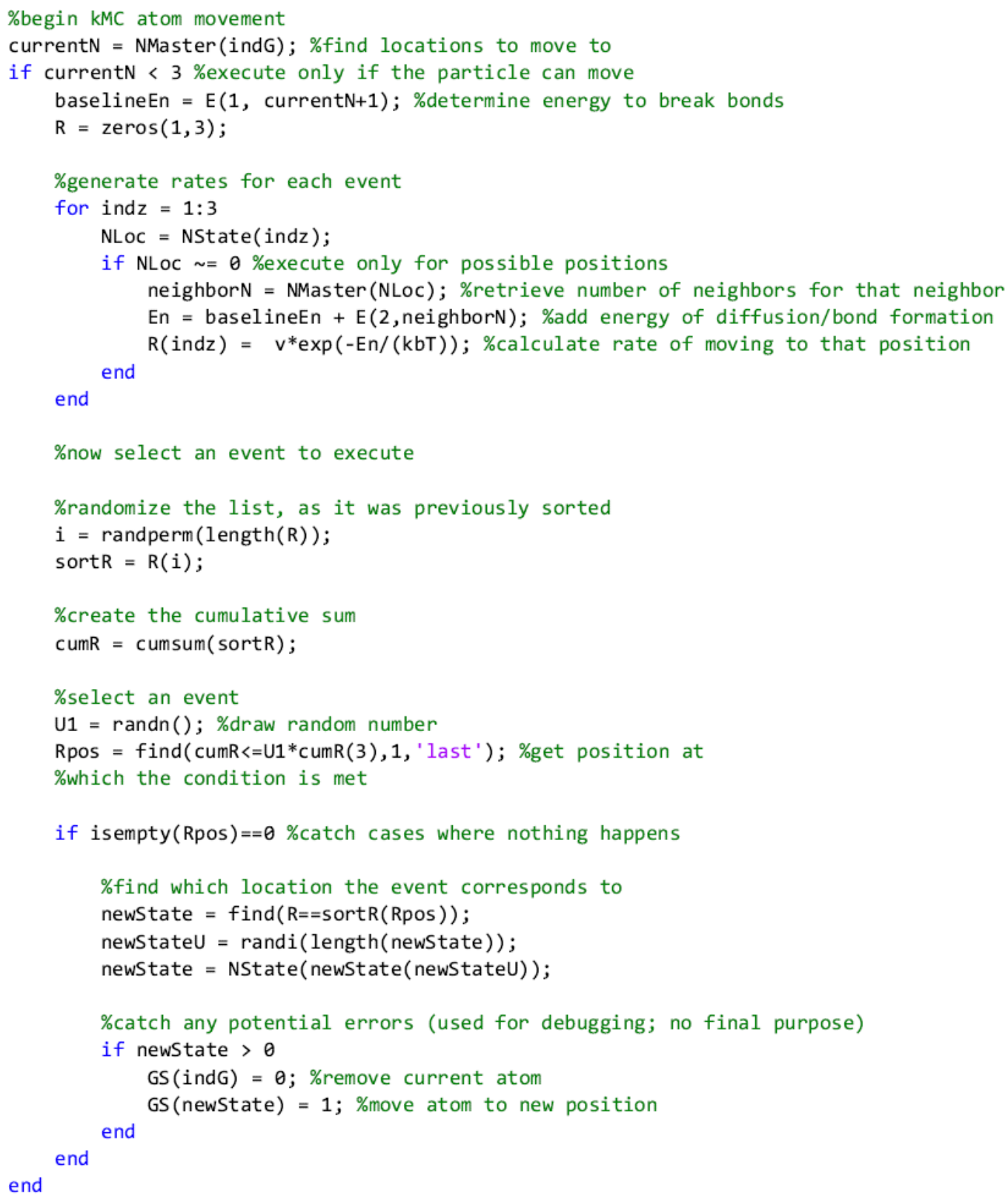

Figure 24. Code that runs the complete kMC movement algorithm, from determining event rates, to selecting an event to execute, to executing said event (as explained in Appendix B).

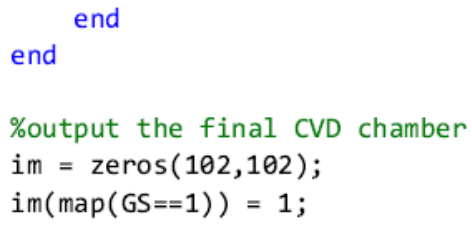

Figure 25. Code that ends open ifs/for loops and outputs the final result for the live script. 


\section{Reprinted Figures (Select Plots Only)}

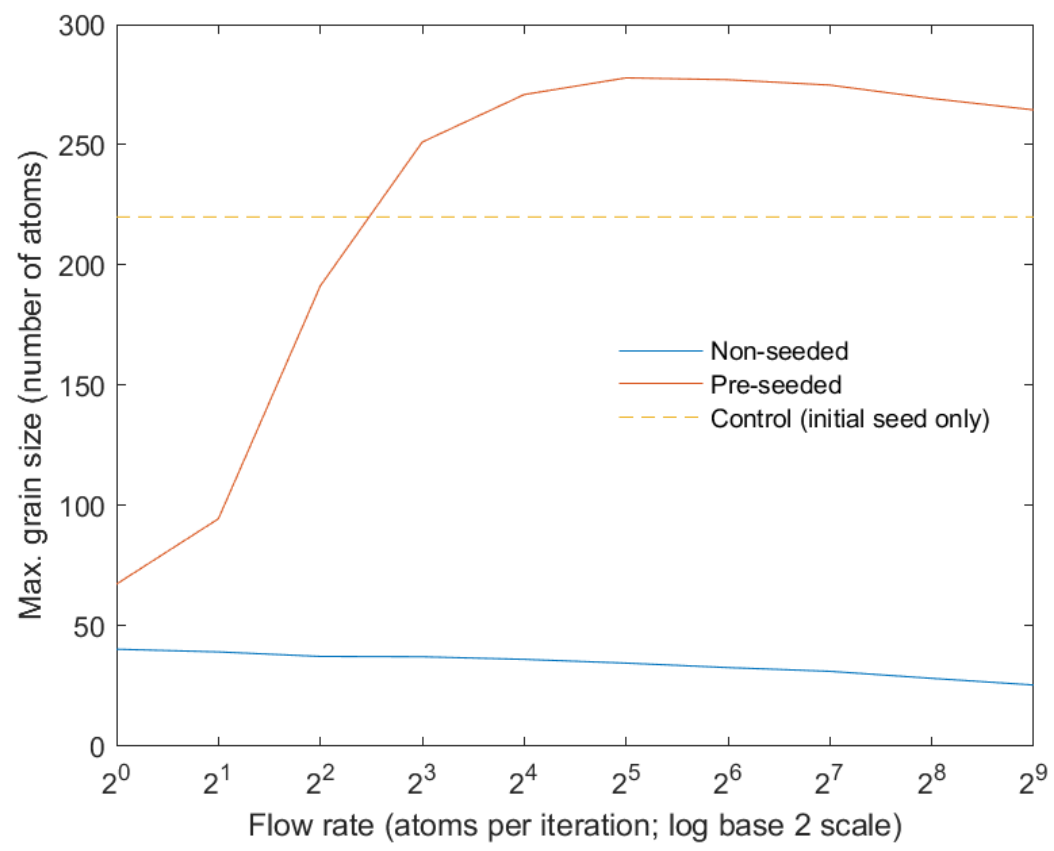

Figure 6. Mean maximum grain size by carbon flow rate, with a control (the atom count of the initial seed) (reprinted from page 4).

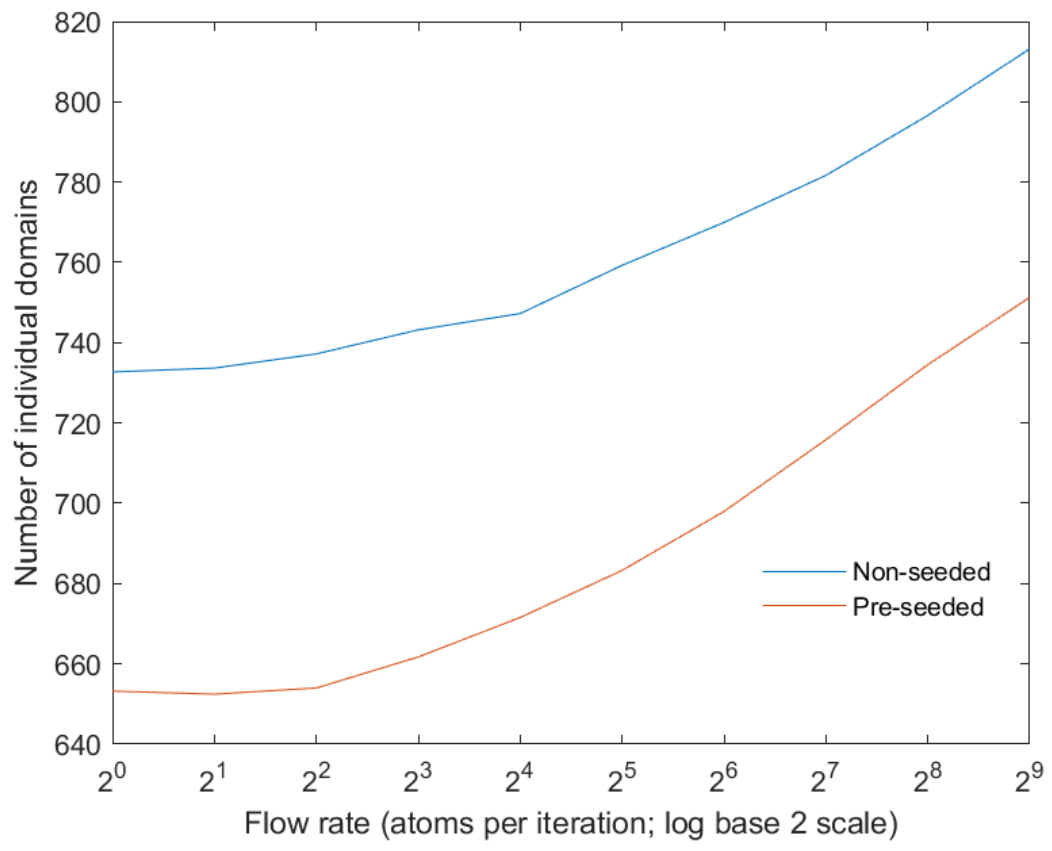

Figure \%. Mean grain count (number of individual grains) by carbon flow rate (reprinted from page 4). 
(a)

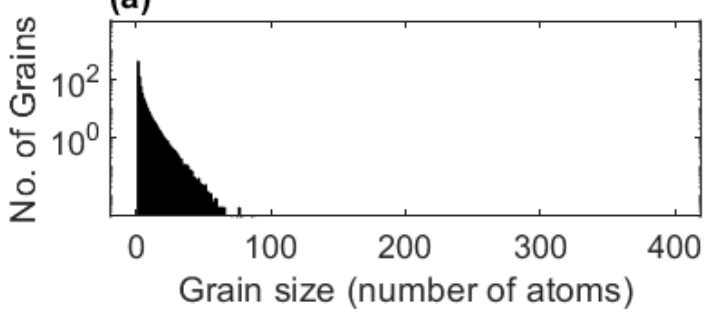

(c)

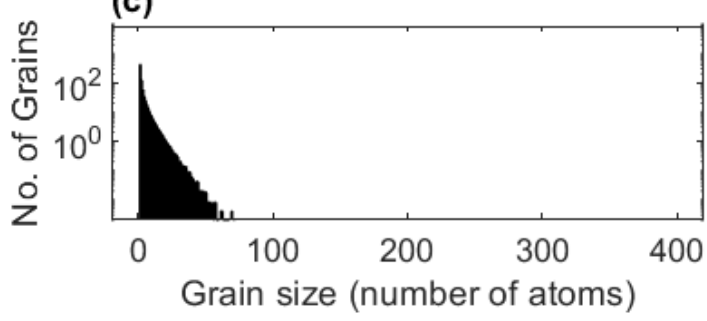

(e)

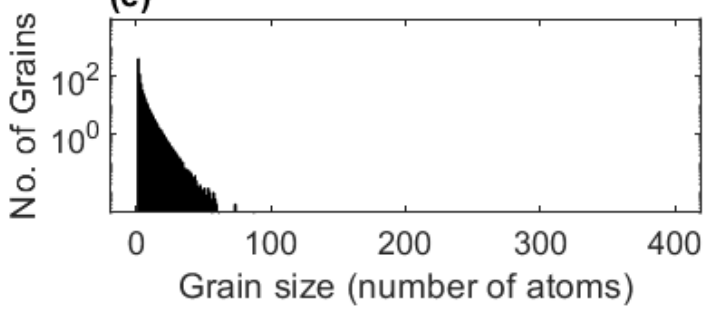

(g)

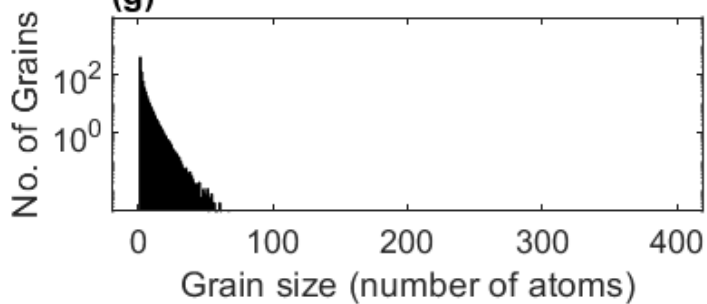

(i)

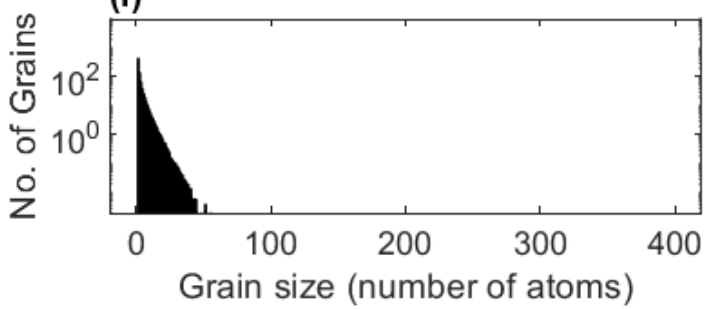

(b)

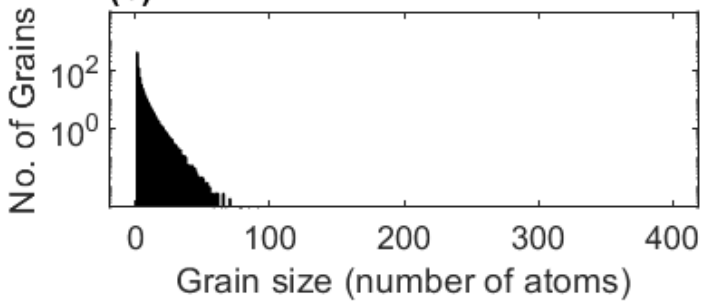

(d)

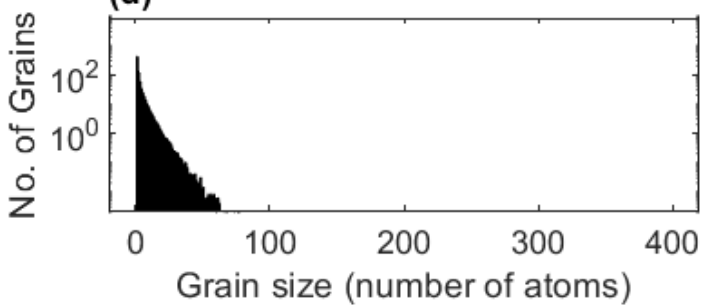

(f)

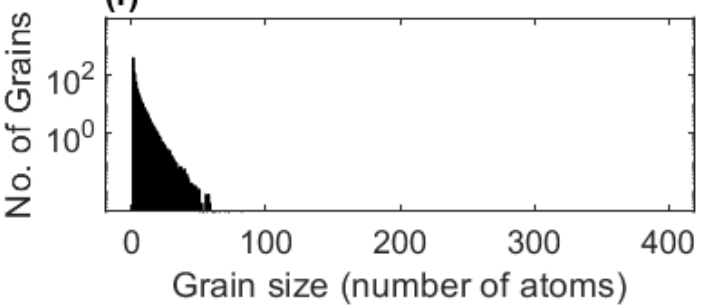

(h)
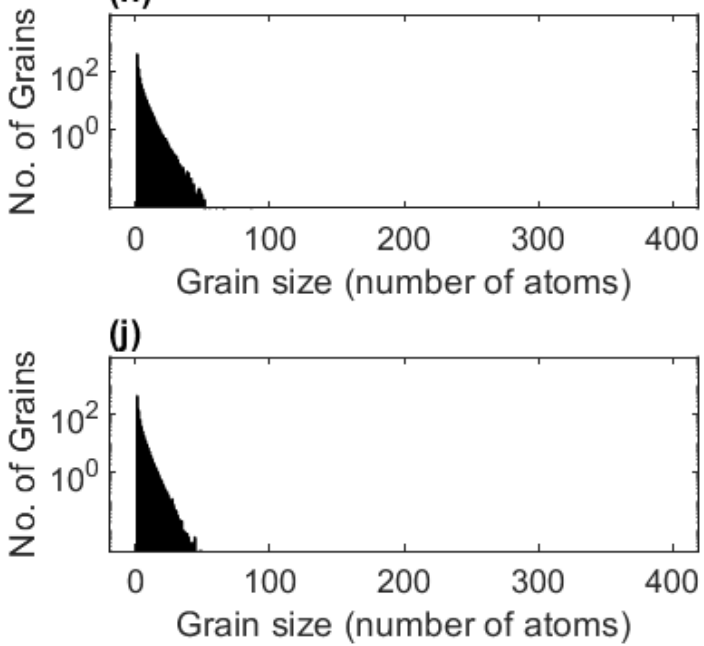

Figure 14. Histogram of mean non-seeded grain size distribution, for flow rates of (a) $2^{0}$, (b) $2^{1}$, (c) $2^{2}$, (d) $2^{3}$, (e) $2^{4}$, (f) $2^{5}$, (g) $2^{6}$, (h) $2^{7}$, (i) $2^{8}$, (j) $2^{9}$ atoms per iteration (reprinted from page 6 ). 
(a)

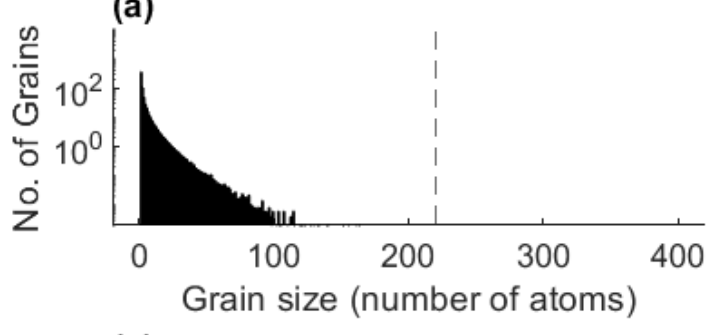

(c)

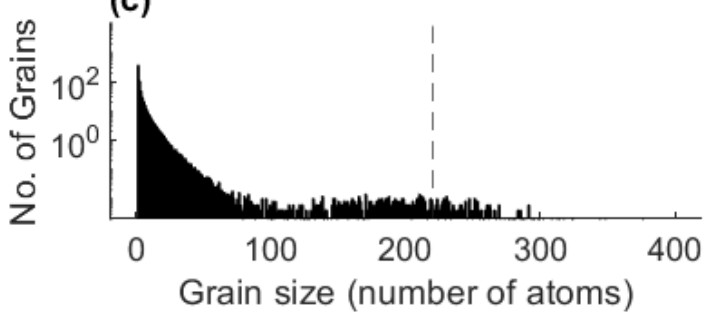

(e)

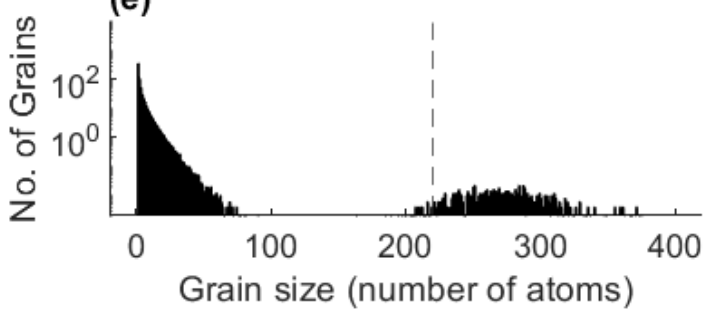

(g)

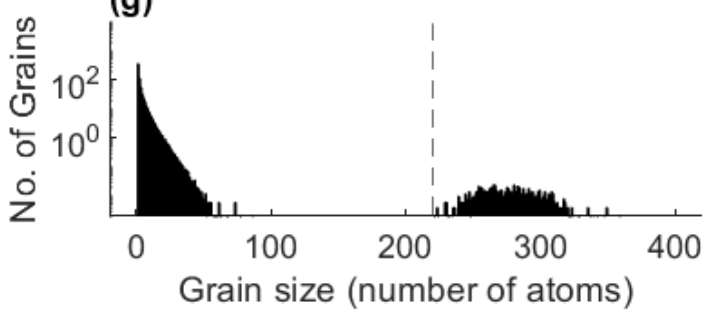

(i)

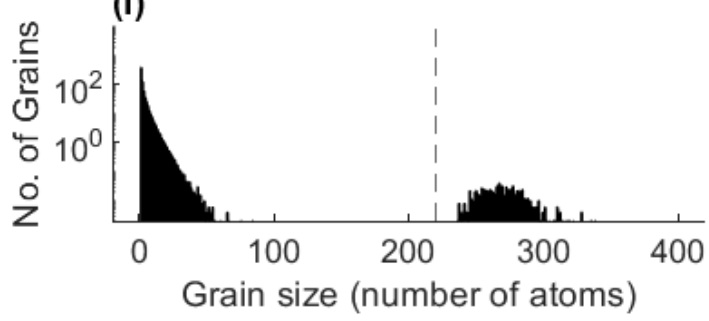

(b)

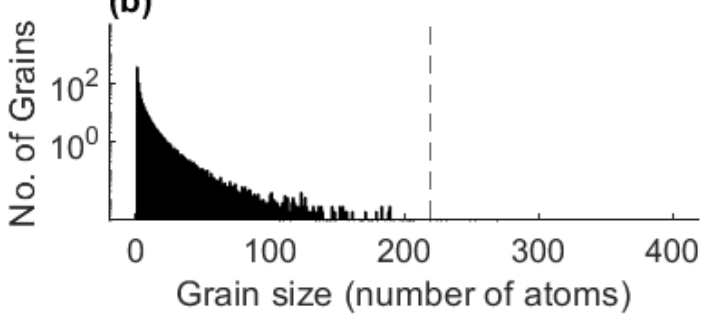

(d)
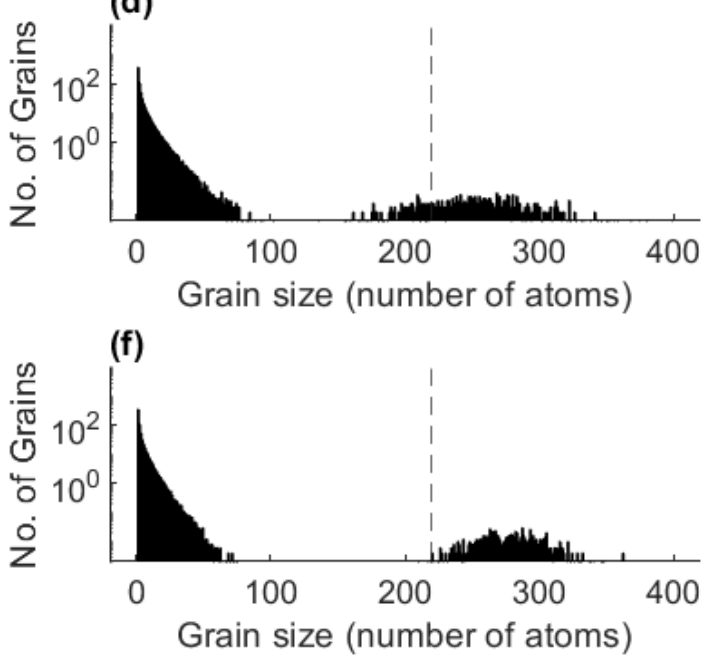

(h)
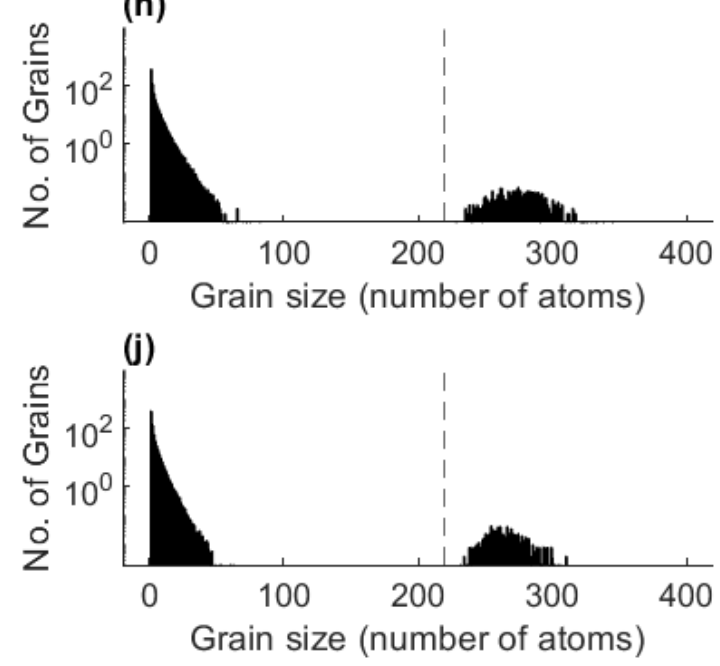

Figure 15. Histogram of mean pre-seeded grain size distribution, for flow rates of (a) $2^{0}$, (b) $2^{1}$, (c) $2^{2}$, (d) $2^{3}$, (e) $2^{4}$, (f) $2^{5}$, (g) $2^{6}$, (h) $2^{7}$, (i) $2^{8}$, (j) $2^{9}$ atoms per iteration. Dashed lines indicate initial pre-seed size (reprinted from page 6 ). 